\title{
Lipid layers on polyelectrolyte multilayer supports $\dagger$
}

\section{Martin Fischlechner, ${ }^{* a}$ Markus Zaulig, ${ }^{a}$ Stefan Meyer, ${ }^{a}$ Irina Estrela-Lopis, ${ }^{a}$ Luis Cuéllar, ${ }^{a}$ Joseba Irigoyen, ${ }^{b}$ Paula Pescador, ${ }^{b}$ Milan Brumen, ${ }^{c}$ Paul Messner, ${ }^{d}$ Sergio Moya ${ }^{b}$ and Edwin Donath ${ }^{a}$}

\author{
Received 7th April 2008, Accepted 30th June 2008 \\ First published as an Advance Article on the web 16th August 2008 \\ DOI: $10.1039 / \mathrm{b805754k}$
}

The mechanism of formation of supported lipid layers from phosphatidylcholine and phosphatidylserine vesicles in solution on polyelectrolyte multilayers was studied by a variety of experimental techniques. The interaction of zwitterionic and acidic lipid vesicles, as well as their mixtures, with polyelectrolyte supports was followed in real time by micro-gravimetry. The fabricated lipid-polyelectrolyte composite structures on top of multilayer coated colloidal particles were characterized by flow cytometry and imaging techniques. Lipid diffusion over the macroscopic scale was quantified by fluorescence recovery after photobleaching, and the diffusion was related to layer connectivity. The phospholipid-polyelectrolyte binding mechanism was investigated by infrared spectroscopy. A strong interaction of polyelectrolyte primary amino groups with phosphate and carboxyl groups of the phospholipids, leading to dehydration, was observed. Long-range electrostatic attraction was proven to be essential for vesicle spreading and rupture. Fusion of lipid patches into a homogeneous bilayer required lateral mobility of the lipids on the polyelectrolyte support. The binding of amino groups to the phosphate group of the zwitterionic lipids was too weak to induce vesicle spreading, but sufficient for strong adsorption. Only the mixture of phosphatidylcholine and phosphatidylserine resulted in the spontaneous formation of bilayers on polyelectrolyte multilayers. The adsorption of phospholipids onto multilayers displaying quarternary ammonium polymers produced a novel 3D lipid polyelectrolyte structure on colloidal particles.

\section{Introduction}

Freestanding lipid membranes, such as lipid vesicles, have numerous applications as model systems for cell membranes and as delivery vehicles. ${ }^{1}$ Lipid layers can also be deposited onto a variety of materials to form what is called a supported membrane. A supported lipid layer provides a means to supply an artificial material with an interface compatible with biological systems and functions. ${ }^{2}$ A typical application of these supported lipid membranes is to serve as a scaffold for membrane-associated biological functions, ${ }^{3-10}$ for example, in our previous work we have used supported lipid layers as a matrix for the attachment of viral functions to colloidal particles via membrane fusion. ${ }^{11}$ The importance of supported membranes from both the scientific and technological point of view has led to extensive research in the past. The formation and properties of supported membranes have been thoroughly elaborated. Indeed, many different solid surfaces have been used as substrates for the formation of supported lipid bilayer membranes (SLBs). ${ }^{12}$

\footnotetext{
anstitute of Medical Physics and Biophysics, Leipzig University, Leipzig, Germany. E-mail: martin.fischlechner@medizin.uni-leipzig.de; Fax. (+49)341-971-5749

${ }^{b}$ CIC biomaGUNE, San Sebastián, Guipúzcoa, Spain

${ }^{c}$ Medical Faculty, University of Maribor, Maribor, Slovenia

${ }^{d}$ Center for NanoBiotechnology, University of Natural Resources and Applied Life Sciences, Vienna, Austria

$\dagger$ Electronic supplementary information (ESI) available: MathCAD program for calculating diffusion constants and calculation of lipid coverage on colloidal particles. See DOI: 10.1039/b805754k
}

A general feature of supported lipid layers is that the lipid membrane experiences an asymmetric environment. The interactions of the lipid layers with the support and with the solution are different. This situation may result in layer properties quite distinct from those of free films composed of the same lipids. In most cases a SLB is formed by the interaction of unilamellar lipid vesicles with the interface. It is widely accepted that the formation of SLBs involves three distinct steps. Vesicles initially attach to the support and then spread into bilayer patches, which have to fuse with one another to finally form a continuous lipid membrane. ${ }^{13}$ For attachment and rupture of the lipid vesicles, their affinity towards the support must be large enough, whereas the formation of the continuous membrane requires local rearrangements of the lipid coverage. Thus, a certain lateral mobility of the lipids along the surface of the support is essential. These somewhat contrary requirements make it very hard to predict whether a genuine SLB will be formed or the attached lipid vesicles will simply arrange themselves in a different topology. The final result strongly depends not only on the quality of the support and the lipids themselves, but also on factors like buffer composition, concentrations and temperature. ${ }^{12,14}$ To date there is a consensus among scientists in the field that despite the relative ease of SLB fabrication on a variety of supports, most of our knowledge on SLB formation and properties remains empirical. This is quite understandable for a number of reasons. The surface of a solid material in contact with an aqueous environment can itself be very complex. It is, for example, difficult to vary the properties of such a surface in a systematic way. The range of techniques applicable for studying the formation and properties of SLBs is limited. There are not too many techniques 
allowing for an in-depth investigation of a thin layer of a few nanometres in thickness on a surface, especially if processes lasting only a few seconds or even fractions of seconds are involved. If soft surfaces such as polyelectrolyte architectures are involved, the dynamics of the surface introduce an additional degree of freedom.

Another point to be considered is that water itself may play an important role in the formation of SLBs. The capability of lipids to form bilayer membranes in aqueous environments is a consequence of the amphiphilic nature of the lipid molecules. Water at the membrane surface is thus a natural component of twodimensional lipid systems. When studying the interaction of the lipid headgroup with the support, effects related to hydration of the support as well as of the headgroup have therefore to be taken into account. Upon SLB formation the hydration state of the support and the lipid layer may change, and this may represent an important contribution to the driving forces of lipid adsorption. For example, the release of "structured" water may increase the entropy of the system as a whole. This scenario has been reported for ion lipid and ion-polyelectrolyte interactions. ${ }^{15-17}$

In our own recent research on biocomposite materials and devices we have employed lipid layers supported by polyelectrolyte multilayers (PEMs). Lipid layers have been used as a means to attach biological functions to colloidal particles and macroscopic surfaces. PEMs were chosen because of their versatility in allowing fabrication of functional composite materials with nanometere precision in the $z$-dimension. A lipid membrane on top of a PEM provides a suitable interface of the artificial polyelectrolyte composite with biological systems, since on one hand it minimizes non-specific interactions and on the other hand it enables the tailoring of the surface with specific interactions through the incorporation of membrane-associated biological functions. ${ }^{18-20}$ For capsules consisting of polyelectrolyte multilayers, the applications of polyelectrolyte supported lipid layers focus on controlling the permeability of the supporting capsule walls. ${ }^{21-24}$

Polyelectrolyte multilayers are a useful system when studying the basic principles of SLB formation. The chemical and physical properties of PEMs are well defined. The nature, arrangement and density of their ionic groups can be varied in a systematic way. Likewise, the lipid composition and electrolyte conditions during coating can be changed. Furthermore, lipid layers can be formed on polyelectrolyte multilayer coated colloidal particles. ${ }^{25-27}$ This brings a considerable advantage over the use of flat macroscopic surfaces because powerful particle-based experimental techniques such as flow cytometry (FACS) can be applied. ${ }^{28}$

The main objective of our work was thus to understand the basic principles of lipid layer formation on top of polyelectrolyte multilayers when these are brought into contact with lipid vesicles in solution. Our specific aims were to identify the binding sites of lipids to the polyelectrolyte interface, to follow the process of layer formation and to understand the underlying driving forces, as well as to clarify the role of small ions in the lipid assembly. The formed lipid layers were investigated with regard to their topology and properties. We explored the behavior of the negatively charged unsaturated phosphatidylserine (1-palmitoyl-2-oleoyl-sn-glycero-3-phospho-L-serine, POPS) and the zwitterionic unsaturated phosphatidylcholine (1-palmitoyl-2-oleoyl-sn-glycero-3-phosphocholine, POPC), as well as of their $1: 1$ mixture, when interacting with multilayers composed of the model polyelectrolytes poly(allylamine hydrochloride) (PAH) or poly(diallyldimethylammonium chloride) (PDADMAC) as examples of weak and strong polycations and poly(styrenesulfonate) (PSS) as a strong polyanion. The top layer of the PEM assembly was varied in a systematic way. Although the choice of this particular set of polyelectrolytes and lipids was motivated by our membrane fusion research, we nevertheless believe that the obtained results may have a more general significance.

\section{Experimental}

\section{Materials}

Silica particles with a diameter of $3 \mu \mathrm{m}$ as well as polystyrene latex beads $20 \mu \mathrm{m}$ in diameter were purchased from microparticles GmbH (Berlin, Germany). Poly(allylamine hydrochloride) (PAH, $70 \mathrm{kDa}$ ), poly(diallyldimethylammonium chloride) (PDADMAC, 20\% w/v, 200-350 kDa), poly(styrenesulfonate, sodium salt) (PSS, $70 \mathrm{kDa}$ ) and phosphate buffered saline (PBS, $10 \mathrm{mM}$ phosphate buffer, $2.7 \mathrm{mM} \mathrm{KCl}, 137 \mathrm{mM} \mathrm{NaCl}, \mathrm{pH} 7.4$ ) were obtained from Aldrich. 1-Palmitoyl-2-oleoyl-sn-glycero3-[phospho-L-serine] (POPS, sodium salt, $10 \mathrm{mg} \mathrm{mL}^{-1}$ in chloroform), 1-palmitoyl-2-oleoyl-sn-glycero-3-phosphocholine (POPC, $10 \mathrm{mg} \mathrm{mL}^{-1}$ in chloroform) and 1,2-dioleoyl-sn-glycero3-phosphoethanolamine- $N$-(lissamine rhodamine B sulfonyl) (PE-rhodamine) were purchased from Avanti Polar Lipids, Inc. Octadecyl rhodamine B chloride (R18) was purchased from Invitrogen.

\section{Methods}

Polyelectrolyte multilayer coating. Silica particles and polystyrene latex particles were coated with 5 or 7 polyelectrolyte layers starting with PAH or PDADMAC. $3 \mathrm{mg} \mathrm{mL}^{-1}$ solutions in $0.5 \mathrm{M} \mathrm{NaCl}$ of PAH, PDADMAC or PSS solutions were used for coating. Adsorption was conducted for $10 \mathrm{~min}$ at room temperature under shaking, followed by 3 centrifugation steps in $0.1 \mathrm{M} \mathrm{NaCl}$.

Formation of the supported lipid membrane. Small unilamellar lipid vesicles consisting of POPS, POPC and their respective mixtures, in PBS or $0.1 \mathrm{M} \mathrm{NaCl}$, were prepared by extrusion through a polycarbonate membrane with a pore diameter of $50 \mathrm{~nm}$ (Avestin Inc., Canada). The lipid bilayer was formed by incubation of the unilamellar vesicles with the multilayer coated colloids for $45 \mathrm{~min}$ in $0.1 \mathrm{M} \mathrm{NaCl}$ or PBS at $37{ }^{\circ} \mathrm{C}$ under continuous stirring. 4 washings with PBS followed. Typically, $50 \mu \mathrm{L}$ liposome solution $\left(5 \mathrm{mg} \mathrm{mL}^{-1}\right)$ was used to coat $25 \mu \mathrm{L}$ of $3 \mu \mathrm{m}$ or $20 \mu \mathrm{m} \mathrm{LbL}$ colloids ( $5 \% \mathrm{v} / \mathrm{v})$ stock solution), respectively. Liposomes labeled with PE-rhodamine were prepared by adding $1 \mathrm{~mol} \%$ to the lipid mixture in chloroform.

QCM-measurements. Quartz sensors were coated with 9 or 11 polyelectrolyte layers adsorbed from polyelectrolyte solutions circulating at a flow rate of $50 \mu \mathrm{L} \mathrm{min}^{-1}$ and at room temperature $\left(25^{\circ} \mathrm{C}\right)$. After equilibration from millipore water to PBS, small unilamellar lipid vesicles in PBS $\left(0.1 \mathrm{mg} \mathrm{mL}^{-1}\right)$ were delivered 
into the measuring chamber. The formation of the lipid membrane was monitored in real time with a Q-sense E4 (qsense, Sweden) with silicon dioxide coated sensors from qsense (QSX 303).

\section{Attenuated total reflection Fourier transform infrared spectroscopy (ATR-FTIR)}

Multilamellar vesicles of POPC and POPS were prepared after drying from a chloroform solution by re-suspending and vortexing several times above and below the main phase transition temperature in $0.1 \mathrm{M} \mathrm{NaCl}$ solutions with $\mathrm{H}_{2} \mathrm{O}$ or $\mathrm{D}_{2} \mathrm{O}$ and the polyelectrolytes, respectively. The final concentration of lipid was $120 \mathrm{~mol} \mathrm{~m}^{-3}$. The polyelectrolyte-to-lipid ratios were $15(\mathrm{~mol}$ $\left.\mathrm{mol}^{-1}\right)$ for PAH and $10\left(\mathrm{~mol} \mathrm{~mol}^{-1}\right)$ for PDADMAC. The vesicle dispersion was applied onto the surface of a ZnSe ATR crystal $\left(70 \times 10 \times 5 \mathrm{~mm}^{3}\right.$ trapezoid, face angle $45^{\circ}$, six active reflections $)$ mounted into a commercial horizontal ATR holder. The hydration degree of the lipid film was adjusted with a moisture generator (HumiVar, Leipzig, Germany) to relative humidity $(\mathrm{RH})$ values between $3 \%$ and $99 \%$ with an accuracy of $\pm 0.5 \%$. Polarized absorbance spectra were recorded with an Excalibur FTS3100 Fourier transform IR spectrometer (Varian, Darmstadt, Germany) at two perpendicular polarizations of the IR beam, parallel and perpendicular with respect to the plane of incidence. IR spectra were measured with a resolution of $4 \mathrm{~cm}^{-1}$ at 100 scans. The peak positions and the center of gravity of the absorption bands were determined from the weighted sum of the polarized absorbancies. ${ }^{29}$

\section{Flow cytometry}

PEM coated silica colloids, $3 \mu \mathrm{m}$ in diameter, were coated with labeled ( $1 \%$ PE-rhodamine) lipid vesicles in $0.1 \mathrm{M} \mathrm{NaCl}$ or PBS respectively. 5 washings in PBS followed. The samples were analyzed by flow cytometry (FACSCalibur, BD Biosciences) with 10000 single colloids measured. The data were analyzed using the WinMDI software written by Joseph Trotter.

\section{Confocal laser scanning microscopy and fluorescence recovery after photobleaching (FRAP)}

Polystyrene latex particles, $20 \mu \mathrm{m}$ in diameter, were coated with 5 to 7 polyelectrolyte layers finishing with PAH, PDADMAC or PSS respectively. Small unilamellar liposomes consisting of POPS, POPC or mixtures thereof including $1 \mathrm{~mol} \%$ PE-rhodamine were used to create the supported lipid membrane. The lipid bilayer was formed by incubation of the unilamellar vesicles, prepared in PBS, with the multilayer coated colloids, in $0.1 \mathrm{M}$ $\mathrm{NaCl}$, for $45 \mathrm{~min}$ at $37^{\circ} \mathrm{C}$ under continuous stirring. 4 washings with PBS followed.

The FRAP experiments were performed with a Leica DM IRBE microscope (lens: HCX PL APO CS, $63 \times$ ). Images were taken at a rate of $1000 \mathrm{~Hz}$ every $0.4 \mathrm{~s}$ with a resolution of $265 \times 256$. TRITC settings (excitation $543 \mathrm{~nm}$, emission 555-620 nm) were used for imaging, with the laser intensity of the $543 \mathrm{~nm}$ Gre/Ne laser set below $20 \%$. Bleaching was achieved by adjusting the intensity of the $\mathrm{Ar} / \mathrm{Kr}$ as well as the Gre/Ne laser to $95 \%$. A bleaching experiment consisted of 20 images taken before bleaching, 100 images during bleaching, followed by 880 images after bleaching. Image stacks

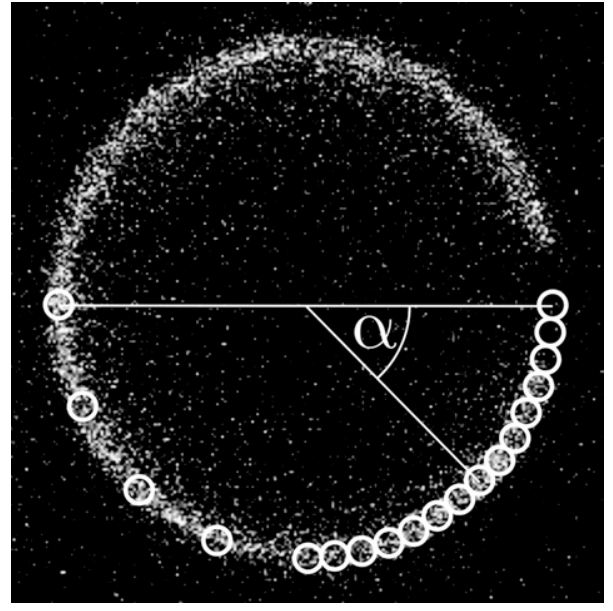

Fig. 1 Confocal image of the equatorial plane of the particle showing the bleaching spot and the circular regions where the intensity was monitored.

were imported to Image J (W. S. Rasband, ImageJ, U. S. National Institutes of Health, Bethesda, Maryland, USA) and the fluorescence intensities of circular regions of interest (see Fig. 1), of about $0.5 \mu \mathrm{m}$ in diameter were obtained using the inbuilt ROI manager. Data was collected in Microsoft Excel and imported to MathCAD 8 (PTC, USA) for further analysis.

Diffusion theory and data analysis. Fig. 1 shows a confocal image of the equatorial plane of a sphere coated with a lipid layer directly after bleaching. The bleach spot at the right side of the sphere appears dark. The angle $\alpha$ denotes the zenith angle in a spherical coordinate system. The fluorescence is symmetric around its azimuth angle. The fluorescence intensity was recorded as a function of time within each of the denoted circles with a diameter of $0.5 \mu \mathrm{m}$ positioned at defined locations on the circumference of the sphere.

Diffusion in spherical coordinates is described by

$$
\frac{\partial c(r, \theta, \varphi, t)}{\partial t}=D \Delta c(r, \theta, \varphi, t)
$$

where $r, \theta$, and $\varphi$ are the spherical coordinates, $D$ denotes the diffusion coefficient and $t$ represents time. Substituting, $x=\cos \theta$, and taking into account the azimuth symmetry, the solution of eqn 1 on the surface of a sphere with radius $r$ can be expressed as a Legendre-Fourier series

$$
I(x, t)=\sum_{i=0} C_{i} P_{i}(x) e^{-\frac{D i(i+1) t}{r^{2}}}
$$

with $C_{n}$ obtained from decomposition of the initial fluorescence intensity distribution, $I(s, 0)$, at $t=0$ :

$$
C_{n}=\frac{2 n+1}{n} \int_{-1}^{1} I(s, 0) P_{n}(s) \mathrm{d} s
$$

$P_{n}$ are Legendre polynomials of order $n$.

The fluorescence recovery measured at different locations was fitted to eqn 2 applying a least square approach. The variable parameters were either the diffusion coefficient together with the 


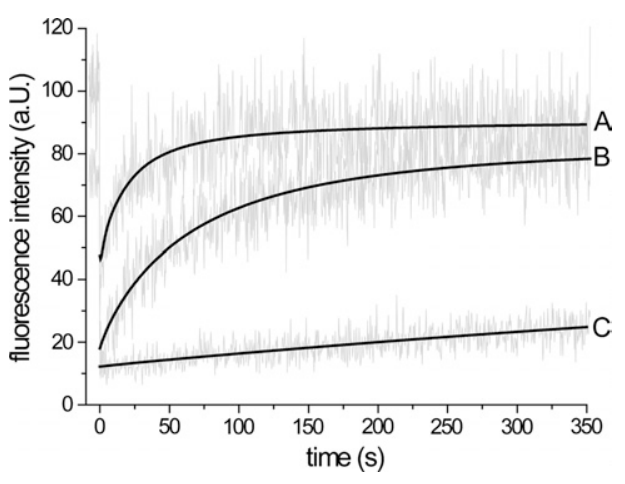

Fig. 2 Original fluorescence recovery data and the respective theoretical curves obtained by fitting. (A) POPS : POPC $(1: 1)$ on PSS/PAH with PAH as the top layer, $\mathrm{D}=3.2 \times 10^{-13} \mathrm{~m}^{2} \mathrm{~s}^{-1}$, recovery $100 \%$; (B) POPS on PSS/PAH with PAH as the top layer, $\mathrm{D}=2.1 \times 10^{-14} \mathrm{~m}^{2} \mathrm{~s}^{-1}$, recovery $100 \%$; (C) POPC on PSS/PAH with PAH as the top layer, D $=2 \times$ $10^{-15} \mathrm{~m}^{2} \mathrm{~s}^{-1}$, recovery $100 \%$.

degree of recovery or two independent diffusion constants according to which the fluorescence recovery was fitted. In this last case the recovery was set to $100 \%$. The coefficient of determination was calculated for each fit and used as a criterion for the reliability of the fit.

Fig. 2 shows typical data for slow, medium and fast recovery together with the obtained fits. The time frame of the experiment allowed for reliable monitoring of the recovery with diffusion constants between $1 \times 10^{-15}$ and $5 \times 10^{-13} \mathrm{~m}^{2} \mathrm{~s}^{-1}$. Since rhodamine derivatives are subject to self quenching, very low label concentrations had to be employed throughout the experiments. This explains the comparatively large fluorescence intensity fluctuations over time as shown in Fig. 2. The short sampling time of $0.4 \mathrm{~s}$ yielded large data sets, which were the basis for the good fits despite the large intensity fluctuations. The intensity recorded in at least three different locations within the bleach spot was used to obtain the diffusion coefficients for every individual experiment. The MathCAD program is available as a text file in the ESI†.

\section{Transmission electron microscopy}

Polystyrene latex particles, $3 \mu \mathrm{m}$ in diameter, were coated with 7 polyelectrolyte layers (PAH/PSS) and decorated with lipid vesicles consisting of phosphatidylserine (PS, $100 \mathrm{~nm}$ in diameter) by incubation in $0.1 \mathrm{M} \mathrm{NaCl}$ followed by 4 washing steps. The colloids were adsorbed onto the microscope grid by placing on it a drop of sample suspension for $1 \mathrm{~min}$. The adsorbed colloids were then negatively stained by transferring the grid to a drop of $1 \%$ aqueous uranyl acetate solution for one minute. Images were taken on a Philips CM12 electron microscope operated at $80 \mathrm{keV}$.

\section{Atomic force microscopy}

Quartz microscopy slides were cleaned by the simplified RCA procedure $\left(\mathrm{NH}_{4} \mathrm{OH}: \mathrm{H}_{2} \mathrm{O}_{2}: \mathrm{H}_{2} \mathrm{O}=1: 1: 5,80{ }^{\circ} \mathrm{C}, 15 \mathrm{~min}\right)$ and coated with 7 layers of PDADMAC/PSS following the same protocol as with the colloids. Lipid vesicles composed of POPS and POPC in a molar ratio of $1: 1$ were applied for lipid layer formation. AFM images in tapping mode were taken using a molecular force probe (MFP, Santa Barbara, CA, USA).

\section{Results and discussion}

\section{Layer formation}

As a result of our empirical efforts to improve the quality of PEM-supported lipid membranes with regard to the integration of viral nanoparticles and the quality of the assembly, we found that a $1: 1$ mixture of POPS and POPC on PAH/PSS with PAH as the top layer showed conspicuously the best performance. While the presence of a certain amount of POPS is mandatory for virus membrane fusion to occur, the purpose of adding POPC was to facilitate the formation of the membrane itself. The choice of unsaturated lipids ensured that at room temperature both lipids were above the main phase transition. The combination of $50 \mathrm{~mol} \%$ POPS and $50 \mathrm{~mol} \%$ POPC yielded a lipid bilayer with the least number of defects, as inferred from a minimum of non-specific interaction together with a comparatively high diffusion coefficient. Details on diffusion will be provided further below.

It was rather surprising that neither POPS nor POPC alone were capable of forming a proper bilayer although both lipids showed considerable adsorption on PAH. The strikingly different behavior between the performance of the components alone and that of the mixture was the starting point of our investigations into the details of lipid bilayer formation on polyelectrolyte multilayers. Our goal was to be able to predict the lipid bilayer assembly on a macroscopic scale through a precise understanding of the interactions taking place at the molecular level.

This starting point of our research is exemplified in Fig. 3. Small unilamellar vesicles (SUVs), prepared in PBS, composed either of only POPC or the negatively charged POPS as well as a $1: 1$ mixture were employed for coating a PSS/PAH multilayer with $\mathrm{PAH}$ as the top layer in a quartz crystal microbalance (QCM) apparatus. The process of lipid bilayer formation was followed in real time. The shift in the resonance frequency of the quartz crystal is a measure of the mass deposited onto the sensor. The changes in dissipation provide information about the structure and softness of the interface. SUVs of the three tested compositions were applied in constant flow to the multilayer coated sensors at room temperature $\left(25^{\circ} \mathrm{C}\right)$. Upon contact of the vesicles with the positively charged surface, all three vesicle preparations readily adsorb on the multilayer. Vesicles composed of the $1: 1$ mixture rupture and spread almost instantaneously after being adsorbed at the surface (Fig. 3B), as can be inferred from the sharp peak indicating a sudden loss of mass just after adsorption has occurred. We attribute this loss of mass to vesicle rupture and the subsequent release of the internal solution. Remarkably, after this quickly proceeding initial phase the mass remained constant regardless of the continued exposition to bulk SUVs, indicating that the bilayer formation was complete. The same sequence of events can be seen in the dissipation behavior. Here the transient sharp peak of dissipation characterizes the initial state where the vesicles become attached, but are not yet completely ruptured and spread. The peak in dissipation is a oneto-one mirror image of the mass change. 


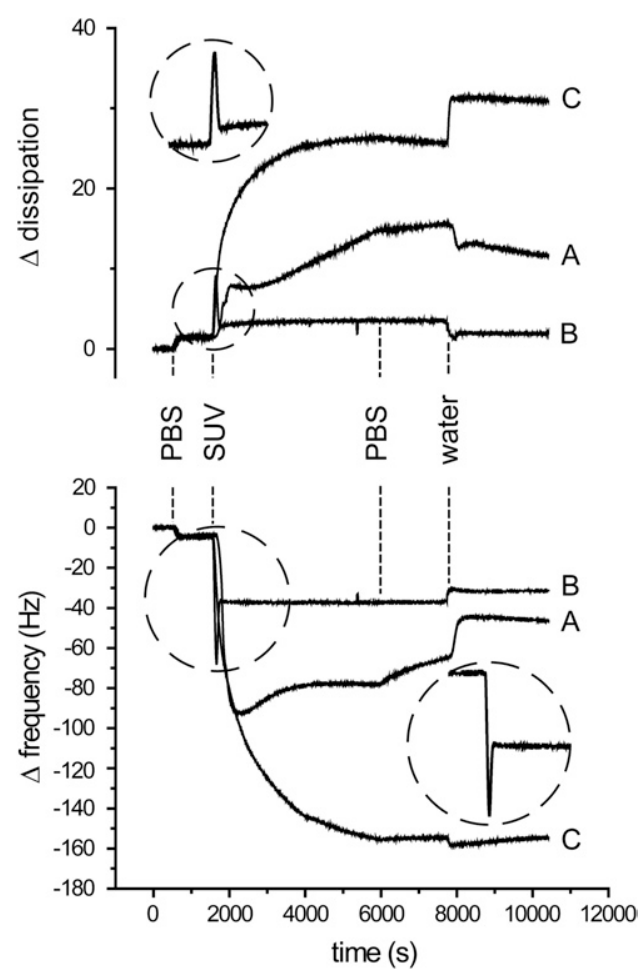

Fig. 3 QCM measurements following SLB formation on PAH. Small unilamellar lipid vesicles composed of POPS (A), 1 : 1 POPS : POPC (B) or POPC (C) were applied in constant flow on multilayer coated sensors. Insets: initial phase of adsorption, rupture and spreading of SUVs consisting of $1: 1$ POPS : POPC.

A quite different behavior was found when vesicles composed of POPS alone (Fig. 3A) were deposited. The adsorption proceeded more gradually, without sharp transient peaks. The adsorbed mass was considerably larger than in the case of the mixture. The initial increase in mass was followed by a slight and gradual decrease, which may be interpreted as either partial rupture of the adsorbed vesicles or as a deformation of the adsorbed vesicles. Eventually a plateau was reached suggesting either completion of adsorption, where all available sites are occupied, or more likely an equilibrium between adsorption and desorption with ongoing vesicle flow. When the vesicle solution is exchanged for buffer, some desorption takes place. Within the time course of the experiment desorption did not terminate. When finally the PBS solution was substituted for water a quick decrease of mass was observed, which can be associated with an osmotically induced rupture of vesicles that are still attached. The corresponding dissipation curve A shows a slow but gradual increase in the interval of constant mass and a plateau in the region of mass loss, indicating structural rearrangements taking place within the adsorbed layer of vesicles. After rinsing in water the dissipation values remained high, although the mass became comparable to that of a bilayer, which can be explained by the presence of osmotically ruptured and collapsed lipid layer fragments. Our conclusion was that the majority of the adsorbed POPS vesicles did not rupture and spread. A proper bilayer was thus not formed. Only after the system was exposed to water might an adsorbed lipid layer together with fragments protruding into the solution have formed. However, this bilayer may not be completely continuous.

POPC vesicles showed a different adsorption behavior (Fig. 3C). The adsorbed amount of lipids increased over almost two hours before a saturation level was reached. The deposited mass exceeded by far that observed for POPS. Rupture and spreading apparently did not take place, as can be deduced from the large increase in dissipation indicating a thick and soft layer at the interface. Upon rinsing in water, a small additional increase in the frequency as well as in the dissipation was attributed to osmotic swelling of intact vesicles adsorbed on the PEM.

\section{Lipid amount and layer topology}

The results of the QCM measurements raise a number of questions. While it is readily understandable that the negatively charged POPS adsorbs on the cationic PAH layer, it is much less clear why this does not result in spreading, rupture, and formation of a proper bilayer structure. Another question refers to the driving forces for the rather high and apparently strong and irreversible adsorption of POPC vesicles on PAH..$^{30}$ From a simple electrostatic point of view, the adsorption of the zwitterionic POPC onto the cationic surface should not be favorable because the counterions of the quarternary ammonium in the choline group and the phosphate group would be confined in an environment with possibly a lower dielectric constant than that of the bulk. Even more mysterious is the quick formation of a seemingly perfect bilayer of $1: 1$ POPS : POPC vesicles on PAH while neither POPS nor POPC alone form a SLB on the same surface.

One of the problems when interpreting QCM data in a quantitative manner is that the resonance frequency shifts include both the mass of the adsorbed lipids and that of the entrapped solution. If lipid vesicles are adsorbed, a considerable fraction of the mass increase could be due to the mass contained within the adsorbed vesicles. The results shown in Fig. 3 thus cannot be directly related to the amount of adsorbed lipid. Therefore, we performed FACS measurements on colloids coated with a PEM, on top of which the lipid layer was formed. The lipid amount can be determined from the fluorescence intensity of labeled PE-rhodamine added at small concentrations to the lipids prior to vesicle preparation. One of the particular advantages of the FACS measurements is that a huge number of single particles are measured in a comparatively short time. Thus, the results not only allow for the determination of the adsorbed lipid amount, but they also provide a measure of the homogeneity of the coating.

Fig. 4 displays the peak maxima of fluorescence intensity of the particles after lipid coating as a function of lipid composition and adsorption protocol for a number of PEM combinations. The top graph shows the adsorption behavior of POPS vesicles. Two observations are immediately apparent: (1) apart from the special case of PDADMAC as the top layer, which showed an extraordinarily large amount of adsorbed lipid and will be discussed further below, the amount of POPS was significantly higher for colloids on which the lipid coating was performed in $\mathrm{NaCl}$ than for those incubated in the presence of phosphate ions. This inhibition of POPS adsorption on PAH by phosphate ions 


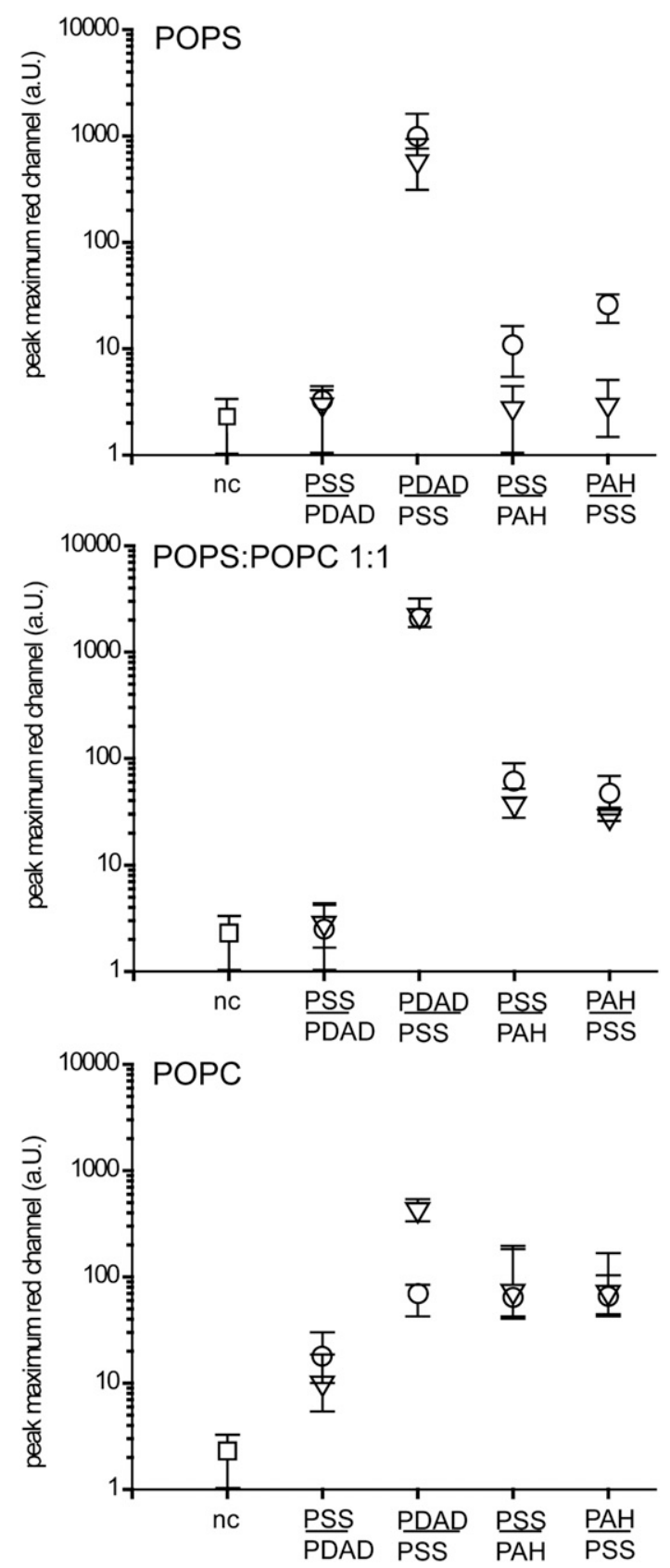

Fig. 4 Quantification of lipid adsorbed on colloidal particles as a function of polyelectrolyte layer sequence, lipid and electrolyte composition ( $\square$ background fluorescence of polyelectrolyte-coated beads, $\bigcirc$ fluorescence of particles coated with vesicles in $0.1 \mathrm{M} \mathrm{NaCl}$ and $\nabla$ with vesicles in PBS; $\perp$ width of the peaks at half maximum height; top polyelectrolyte layers are underlined).

strongly suggests a competitive binding of phosphate and POPS to the amino group of PAH.

(2) Binding of the negatively charged POPS was observed even if PAH was not the top layer, but was located underneath a top PSS layer. Contrarily, adsorption of POPS did not occur if PDADMAC was underneath PSS. These observations together indicate that POPS binds to PAH and that in the case of PSS as the top layer some PAH chains are nevertheless available for binding to the incoming POPS vesicles. Binding of the highly negatively charged POPS to the likewise highly negatively charged PSS, although in principle possible via counterion correlation, ${ }^{31}$ can be ruled out, since no binding is observed when PDADMAC is covered by a PSS layer.

The lower graph in Fig. 4 corresponds to the adsorption of POPC. Apart from the binding behavior in the presence of PDADMAC, three observations are the key to understanding the process: (1) phosphate ions do not inhibit the binding of POPC vesicles to PAH, as was the case with POPS. (2) Regardless of whether the top layer was PAH or PSS with PAH located underneath, roughly similar quantities of POPC were adsorbed. (3) Binding to PSS with PDADMAC underneath was weak in terms of the amount of adsorbed lipid. These observations taken together allow us to conclude that POPC binds strongly to PAH and that this binding cannot be inhibited by phosphate ions. The binding mechanism of POPC to PAH thus differs substantially from that of POPS to PAH.

The middle graph in Fig. 4 shows the adsorption of the $1: 1$ POPS : POPC mixture. Again, an extraordinarily large amount of lipid was adsorbed on the PDADMAC/PSS composite when PDADMAC was the top layer. However, when PSS was on top of PDADMAC, almost no adsorption occurred. Concerning the binding of the $1: 1$ POPS : POPC mixture to the PAH/PSS system the main observations may be summarized as follows: (1) regardless of whether PAH or PSS constituted the top layer, a similarly large amount of lipid was adsorbed on the multilayer. When comparing the adsorption of the $1: 1$ mixture with that of pure POPC it can be noticed that the adsorbed amount was significantly smaller for the mixture. This difference in the bound quantities is more readily evident from the histogram data presented in Fig. 5. (2) Phosphate ions did not have a pronounced influence on the binding behavior of $1: 1$ POPS : POPC.
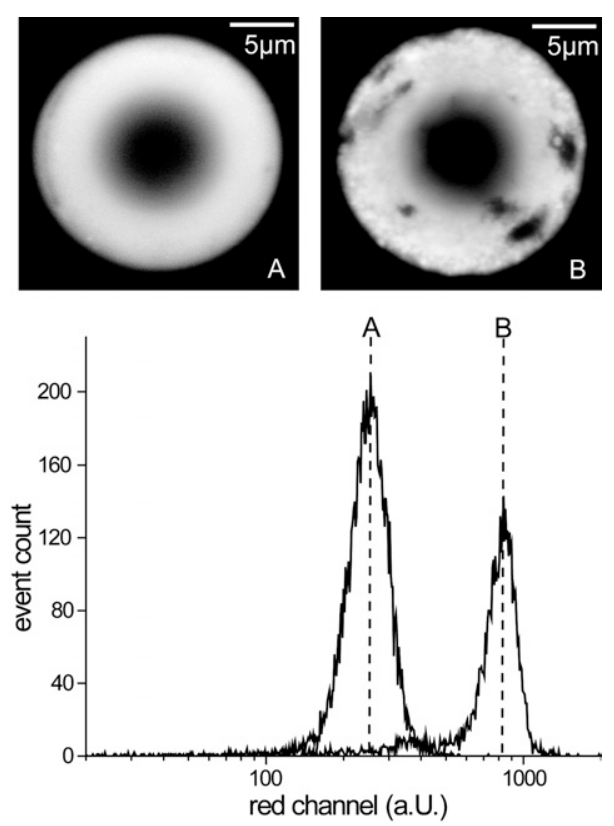

Fig. 5 3D-scans of lipid coated colloids, $20 \mu \mathrm{m}$ in diameter. (A) $1: 1$ POPS : POPC, (B) POPC. Flow cytometry histograms of single colloids coated with $1: 1$ POPS : POPC (A) and POPC (B). 
From these results it follows that the binding behavior of the POPS : POPC mixture on the PAH/PSS system was determined by POPC. This conclusion comes specifically from the absence of a pronounced effect of phosphate ions, as opposed to the case of POPS alone, and from the intense adsorption of the negatively charged vesicles to the negatively charged PEM system, which was not observed when POPS was the only adsorbing lipid (top graph in Fig. 4). Although POPC determined the binding behavior of the mixture, this does not necessarily mean that POPS did not have an important influence on the properties of the composite system. The specific role of POPS will be explored now in more detail.

Fig. 5 shows confocal microscopy 3D-scans of the surface of colloids carrying a PSS/PAH multilayer with $\mathrm{PAH}$ as the top layer, on which lipid vesicles with a fluorescent lipid probe have been adsorbed. The central part of the images appears nonfluorescent because that area of the colloid is very close to the support. The exciting laser beam is subject to reflections in the narrow gap between the particle and the support, and the subsequent interference leads to fluorescence intensity distortions in this area. Therefore, the central region was excluded from the analysis.

The left image (a) corresponds to a colloid coated with the $1: 1$ mixture of POPS and POPC, while image (b) was obtained after coating with POPC vesicles. A striking difference can be immediately appreciated. Image (a) shows a homogeneous coating, whereas image (b) is characterized by some relatively large areas with missing lipid coverage and by a non-homogeneous fluorescence on a scale below $1 \mu \mathrm{m}$. The rim of the right image shows two apparent distortions from sphericity at 9 and between 11 and 12 o'clock. These defects indicate that the lipid coverage has a pronounced extension into the radial direction. The conclusion was that in the case of POPC alone, the vesicles adsorb readily but do not rupture and spread, and hence they do not form a continuous bilayer, but rather a layer of vesicles. Apart from some defects, which might have been induced by the intense washing, vesicles cover the colloidal particle surface.

This supposition is further confirmed by the flow cytometry histograms displayed below the respective confocal images. The difference in the amount of adsorbed lipids between $1: 1$ POPS : POPC on one hand and POPC on the other hand is obvious, and it can be explained by the different topologies of the lipid arrangement. Straightforward geometrical considerations demonstrate that a sphere completely covered with much smaller vesicles would have $4 \pi / \sqrt{ } 12 \cong 3.6$ times more lipid on its surface than a sphere covered with an adjacent bilayer (see the ESI $\dagger$ ). The ratio between the two geometric mean fluorescence intensities displayed in the two histograms in Fig. 5 was about 3.5, very close to the theoretically expected value. The excess of POPC over a bilayer coverage varied somewhat from experiment to experiment, ranging from about a factor of 1.5 to values close to the theoretical maximum of 3.6. This result is in good agreement with the QCM measurements, which showed the existence of a layer formation process for the POPS : POPC mixture, but only adhesion kinetics in the case of POPC vesicles.

Looking at the behavior of POPS vesicles in contact with the PAH surface, the QCM measurements did not provide an unambiguous picture of the processes taking place upon interaction. Although there might be some rupture and spreading of

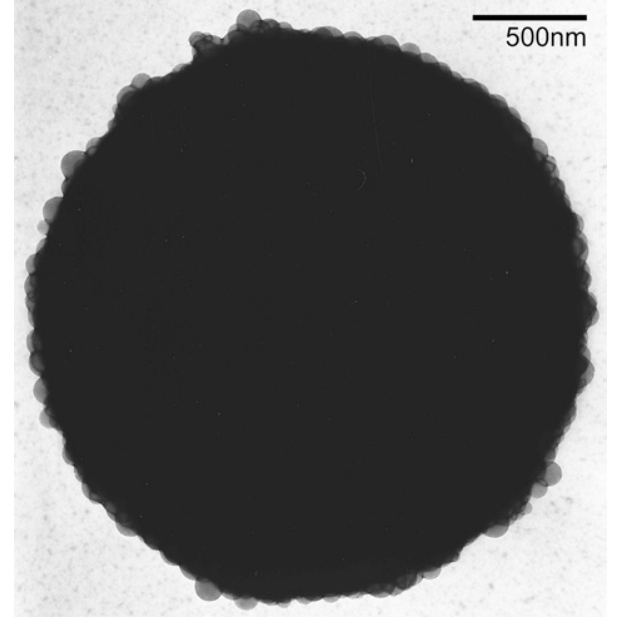

Fig. 6 TEM image of a negatively stained $3 \mu \mathrm{m}$ sized particle coated with PS vesicles $100 \mathrm{~nm}$ in size.

the adsorbed vesicles, the comparatively large mass increment seen in curve A of Fig. 3 together with the increasing dissipation prove that a considerable fraction of the adsorbed vesicles remained intact and therefore did not release their content.

This conclusion is also supported by TEM images. Fig. 6 shows a dense layer of PS vesicles that have adsorbed onto the PAH top layer in $0.1 \mathrm{M} \mathrm{NaCl}$. The majority of vesicles appear flattened, and vesicle-vesicle contacts can be identified. Confocal microscopy scans of such colloids reveal within the resolution of the instrument a homogeneous lipid layer, although lipid fragments, which cannot be removed by thorough washings, can still be appreciated. In contrast, TEM images of colloids coated with a 3 : 1 mixture of PS and PC do not show vesicles, but a smooth lipid layer. ${ }^{32}$

The main findings obtained so far can be summarized as follows: only when a mixture of POPS and POPC was employed did a lipid bilayer spontaneously form on a PEM system with $\mathrm{PAH}$ as the top layer. In the case of the pure lipids, either POPS or POPC, vesicles readily adsorbed and formed a dense layer on the surface of the colloid, but the transition toward a bilayer structure did not proceed. The ionic composition of the surrounding electrolyte was an important factor influencing the interaction of POPS with PAH. In the presence of phosphate buffered saline (10 $\mathrm{mM}$ phosphate, $137 \mathrm{mM} \mathrm{NaCl})$ the interaction of POPS with PAH was weaker than in the presence of chloride ions, but both conditions led to incomplete rupture and spreading of adsorbed vesicles. The ionic composition did not however affect the interaction of POPC with PAH. $\zeta$ potential measurements showed that the positive charge of PEM with $\mathrm{PAH}$ as the top layer disappeared at low concentrations of phosphate buffer and the colloidal particles became negative. Indeed, for $400 \mathrm{~nm}$ silica particles coated with 5 layers PAH/PSS the $\zeta$ potential became negative at $40 \mathrm{mM}$ phosphate with a value of $-6.60 \mathrm{mV}$. For particles coated with 10 layers the change of sign takes place at a lower ionic strength, displaying a $\zeta$ potential of $-4 \mathrm{mV}$ at $20 \mathrm{mM}$ phosphate buffer. Electrophoretic measurements performed at higher concentrations $(60,80,100$ and $200 \mathrm{mM}$ phosphate) resulted in negative values of $\zeta$ potential. Control experiments in $\mathrm{NaCl}$ for the same ionic strengths 
gave positive values of $\zeta$ potential for all the considered concentrations. These results show that there is a specific interaction of the phosphate ions with the amino groups in PAH. The $\zeta$ potential of colloids with PDADMAC as the top layer was not affected by phosphate ions. The nature of the interaction of phosphate groups with amines will be analyzed later in the paper.

\section{Specificity of the interaction of PDADMAC/PSS multilayers with lipid vesicles}

Fig. 4 shows that the amount of $1: 1$ POPS : POPC adsorbed on a colloidal particle with PDADMAC as the top layer was about 50 times larger than that necessary to form a bilayer. Confocal images (not shown) revealed a homogeneous lipid coverage with the expected intense fluorescence. The scattering data taken in parallel with the fluorescence recordings in the flow cytometry experiments showed, in view of the overall increase in the adsorbed mass, a rather surprising decrease of the sideward scattering intensity. The latter observation can only be explained by the coating becoming optically more homogeneous after interaction with the lipid, resulting in smaller refractive index differences. This evidence indicates that the lipids did not just adsorb onto the PEM structure but most likely formed a 3D lipid polyelectrolyte composite structure, perhaps by integration into the multilayer. Scanning force experiments were conducted to shed some light on the nature of the fabricated polyelectrolyte lipid composite structure. Fig. 7 shows the images obtained. The surface of the composite is characterized by numerous pores ranging in diameter over one order of magnitude, from 50 to $500 \mathrm{~nm}$, and bullous structures appear underneath the surface as inferred from the slightly different softness showing up in the phase image.

This was an intriguing observation. The composite showed a sponge-like structure which maybe represented a polyelectrolyte supported, spontaneously assembled 3D-network of lipid lamellae. Similar structures are known to occur in solution
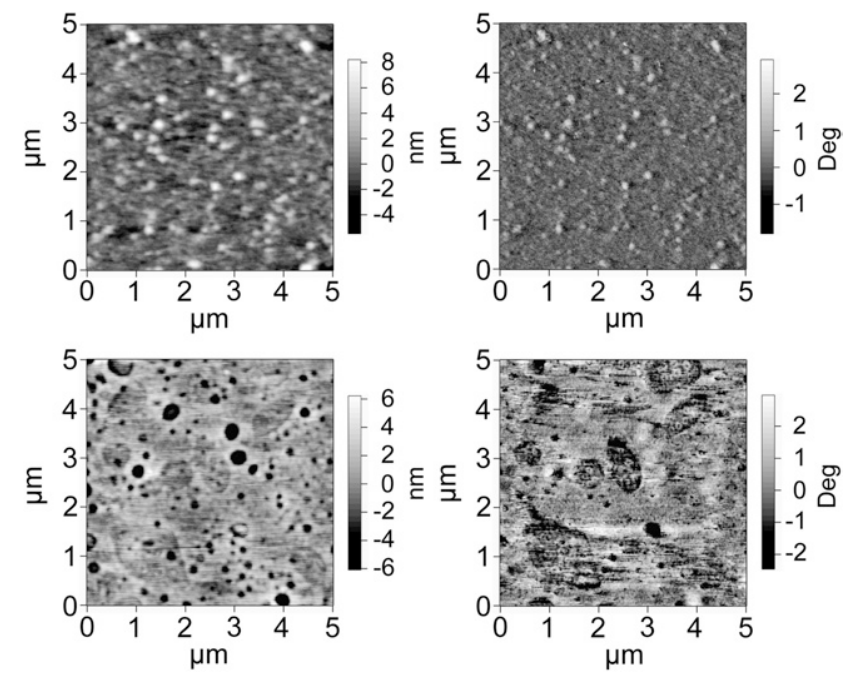

Fig. 7 Top-view (left) and phase (right) scanning force images of a PSS/ PDADMAC polyelectrolyte multilayer with PDADMAC as the top layer before (top images) and after incubation with $1: 1$ POPS : POPC (bottom images). upon spontaneous complexation of lipids or other surfactants with polyelectrolytes. ${ }^{33-35}$ To the best of our knowledge, such a structure forming the outer layer on a colloidal composite fabricated by exposing a polyelectrolyte multilayer to lipid vesicles has not been reported previously. This finding certainly deserves further investigations towards resolving the structure and exploring its properties as well as possible applications.

\section{Lipid diffusion and layer connectivity}

The lateral mobility of lipids in supported lamellar phases is an important parameter characterizing the strength of their interaction with the support. Since POPS and POPC are well above their phase transitions at room temperature, a decrease in their lateral diffusion compared to freestanding films can be directly related to attractive interactions with the support. We performed FRAP experiments where lipid diffusion and fluorescence recovery on the surface of the colloid were followed over lateral separations of several micrometres. ${ }^{36-38}$ Diffusive fluorescence recovery over these comparatively large distances thus not only requires lipid mobility, but also connectivity of the lipid film on the scale of $1 \times 10^{1} \mu \mathrm{m}$.

Fig. 8 displays the results of a systematic study of lipid diffusion on the colloidal spheres as a function of the composition of the vesicles employed for the SLB formation. The lipid composition ranged from pure POPC to pure POPS including different ratios of both components.

Diffusion of the PE-rhodamine probe was negligibly small, D $\approx 1 \times 10^{-15} \mathrm{~m}^{2} \mathrm{~s}^{-1}$, in the case of POPC adsorbed on PAH. The diffusion coefficient of PE-rhodamine in POPS adsorbed on PAH was one order of magnitude larger, but still very small (two orders of magnitude slower) compared to the typical values reported for free films. ${ }^{39}$ The POPS/POPC mixtures revealed a markedly different behavior. These layers were characterized by a pronounced fluorescence recovery and correspondingly showed comparatively large diffusion coefficients. The $1: 1$ mixture showed a maximum diffusion coefficient of about $3.8 \times$ $10^{-13} \mathrm{~m}^{2} \mathrm{~s}^{-1}$, while the diffusion coefficients decreased when any of the components was in excess. The best results with respect to the homogeneity of lipid diffusion in sample preparations were achieved by incubating colloids in $0.1 \mathrm{M} \mathrm{NaCl}$ with lipid vesicles prepared in PBS buffer.

The striking difference in the apparent mobility between the pure lipid and the mixture are most likely related to differences in

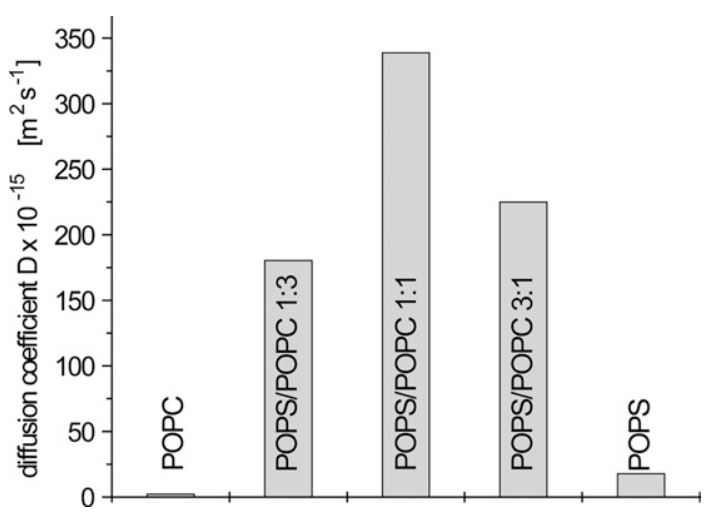

Fig. 8 Diffusion coefficients of PE-rhodamine on PAH. 
film topology. We interpret the apparent absence of mobility in the case of POPC and the very low mobility in the case of POPS as the result of missing or insufficient connectivity of the lipid film. This is fully consistent with the conclusions drawn from the flow cytometry and confocal microscopy experiments. While the QCM data alone did not allow unambiguous conclusions to be drawn about the presence or the absence of a continuous film underneath the adsorbed vesicles, in view of the rather low mobility it seems highly unlikely that POPS forms such a uniform layer on PAH.

Lipid diffusion may be quite asymmetric for lipid membranes on top of PEM. The diffusion in the monolayer in contact with the support may be quite different from diffusion in that half of the film that is exposed to the bulk. ${ }^{40}$ This has to be kept in mind when referring to quantitative aspects of diffusion. Since there is a strong attractive interaction of both POPS and POPC to PAH, lipid diffusion in the lower half of the film may be strongly inhibited. To actually measure the diffusion in lipid layers in contact with a polyelectrolyte support, the following experiment was conducted. Lipid layers were intercalated between polyelectrolyte films by adsorbing additional PE layers on top of the deposited lipid layer. ${ }^{41,42}$ The diffusion coefficients in intercalated lipid layers are shown in Fig. 9. Regardless of whether POPS or the mixture was used for the intercalation between PAH layers, a strong decrease of the diffusion coefficient down to approximately $3 \times 10^{-15} \mathrm{~m}^{2} \mathrm{~s}^{-1}$ was observed. Our conclusion was that the strong interaction with the polyelectrolyte was responsible for the apparent immobilization of the lipid molecules. In view of this result we assume that in our system only those lipids facing the bulk but not those oriented toward the support contributed to diffusion. In such a situation the recovery should be theoretically far from complete because roughly only $50 \%$ of the lipids are mobile. However, we could not find convincing experimental evidence for an incomplete recovery despite a careful analysis of the recovery behavior. Although in some cases two processes with different rates could be identified, the experimental fluctuations in intensity were too large compared with the systematic variations of the intensity and did not allow for precise discrimination between processes with different rate constants. In some experiments we used $\mathrm{R} 18^{43}$ instead of labeled $\mathrm{PE}$ as the fluorescent marker. In these experiments we indeed found good

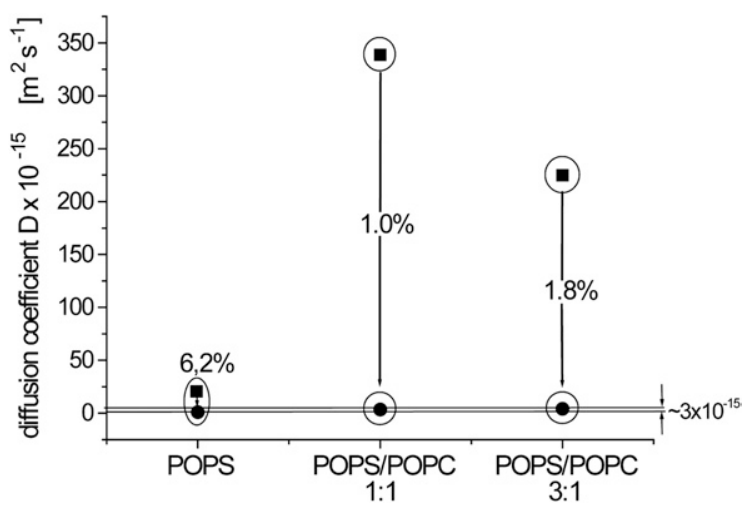

Fig. 9 Reduction of diffusion coefficients by additional polyelectrolyte layers added on top of the lipid layer ( $\mathbf{\square}$ lipid as top layer, $\bullet$ lipid covered with 4 additional PE layers).

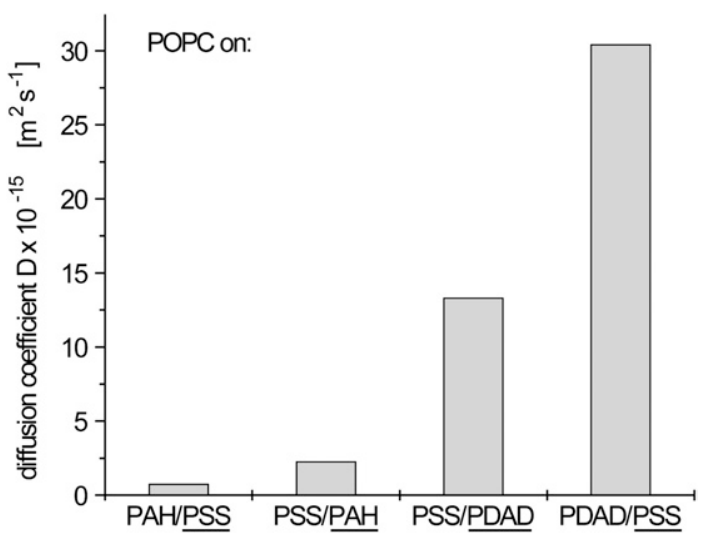

Fig. 10 Changes in lipid diffusion coefficients of membranes composed of POPC depending on the polyelectrolyte support (top polyelectrolyte layers are underlined).

evidence for a recovery to only half of the maximum value. The single reasonable possibility to explain the complete recovery together with the missing lateral mobility in the lower half of the bilayer was to assume a trans-bilayer movement of the label. In terms of rate constants, this flip-flop has to be comparable with lateral diffusion. The existence of such a trans-bilayer movement does not seem unlikely, because the lipid film is probably under considerable stress due to its strong and asymmetric interaction with the support. ${ }^{44}$ This situation would be consistent with the existence of membrane defects. ${ }^{45}$ It also must be taken into account that the headgroup of the label contains the bulky and less water soluble fluorophore. Furthermore, the hydrocarbon phase of the film is less ordered because the lipids contain unsaturated fatty acids. All these factors may lead to an enhanced probability of trans-bilayer movement of the label.

Fig. 10 compares the diffusion behavior of PE-rhodamine in POPC on PDADMAC/PSS with the PAH/PSS PEM system. The graph shows that both for PAH as the top layer or located underneath PSS, a continuous lipid layer over distances of the order of $1 \times 10^{1} \mu \mathrm{m}$ is not formed. However, when PAH was substituted by PDADMAC some diffusion was observed. These data have to be compared with the results displayed in Fig. 4. In the case of PSS with PDADMAC underneath, the adsorbed lipid amount is not sufficient to form a bilayer over the entire surface of the particle. The low diffusion constant, of about $1.3 \times 10^{-14}$ $\mathrm{m}^{2} \mathrm{~s}^{-1}$, reflects thus the limited connectivity of the lipid layer. In the case of PDADMAC as the top layer the quantity of adsorbed lipid is much larger and exceeds by many times the amount necessary to form the bilayer. The measured diffusion constant may be related to lipid diffusion in a complex 3D-topology.

\section{Identifying the binding sites and driving forces}

ATR-FTIR was employed to study the interaction of POPC and POPS with the respective polyelectrolytes. The influence of the hydration state and lipid conformation on the infrared spectral characteristics of phospholipids is well known..$^{29,46-54}$ The spectra of the pure phospholipid can thus be used as a reference when comparing lipid spectra in the absence and presence of the respective polyelectrolyte species. If there are characteristic 
differences, conclusions about possible binding sites and the effect of the binding polyelectrolyte on the lipids may be drawn.

Only the cationic polyelectrolyte species induced typical changes of the IR spectrum of the phospholipids. In the presence of PSS we did not find pronounced deviations of the spectrum, which could have been attributed to an interaction with the phospholipids on the molecular level. Therefore, we concluded that a specific interaction of PSS with POPC or POPS is unlikely. The situation was, however, quite different for each of the two cationic polyelectrolyte species employed.

\section{POPC-PAH}

Fig. 11 shows a section of the fingerprint region of the IR absorption spectrum of POPC ranging from $1320 \mathrm{~cm}^{-1}$ to 1030 $\mathrm{cm}^{-1}$ in the absence (curve 1) and presence (curve 2) of PAH at high and low hydration, together with the PAH spectrum as a control (curve 3). Let us start with a brief description of the control POPC spectrum and the relevant changes occurring upon changes in the hydration state. The presented section covers the "phosphate region", including the asymmetric stretch of phosphate $\left(\mathrm{PO}_{2}^{-}\right)$characterized by two components at $v_{\text {as }}=1220$ $\mathrm{cm}^{-1}$ and $v_{\mathrm{as}}=1229 \mathrm{~cm}^{-1}$, as well as the symmetric stretch at $v_{\mathrm{s}}=$ $1087 \mathrm{~cm}^{-1}$. These absorption bands refer to the hydrated state. Upon dehydration the frequency of the asymmetric $\mathrm{PO}_{2}{ }^{-}$stretch vibration increases to approximately $1252 \mathrm{~cm}^{-1}$. In the dehydrated state there are wagging peaks superimposed onto the

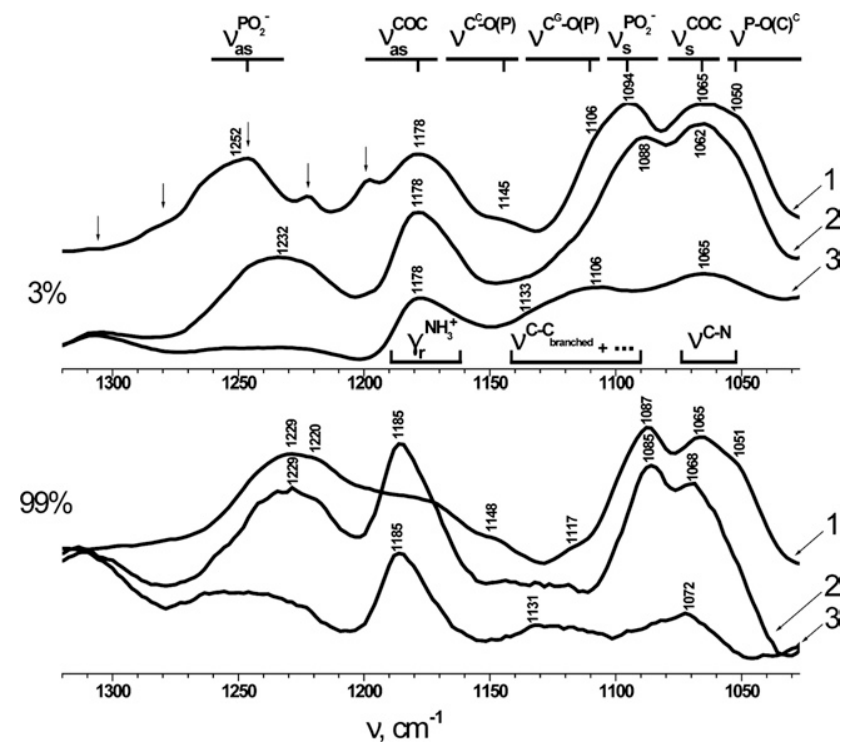

Fig. 11 ATR-FTIR fingerprint region spectra of hydrated (bottom) and dehydrated (top) POPC (curve 1) and POPC with PAH (curve 2) together with the PAH control (curve 3). The assignment of the lipid bands is given at the top of the spectrum. The assignment of PAH bands is shown below the PAH spectrum. ${ }^{54}$ Arrows indicate the wagging progressions of dehydrated POPC chains. $v_{\mathrm{s}}$ and $v_{\mathrm{as}}$ refer to symmetric and asymmetric stretching vibrations. $\gamma_{\mathrm{r}}$ indicates rocking. The stretching PAH bands centered at $1133 \mathrm{~cm}^{-1}$ and $1106 \mathrm{~cm}^{-1}$ represent combinations of $\mathrm{C}-\mathrm{C}$ stretching of the branch with motion of the side chains. The spectra referring to pure POPC and POPC with $\mathrm{PAH}$ were standardized taking the intensity of the phosphate vibrations as reference. The intensity of the $\mathrm{PAH}$ control spectrum is normalized with the POPC-PAH spectrum taking the $\mathrm{C}-\mathrm{C}-\mathrm{N}$ vibrational band intensity as a reference. phosphate band giving rise to peak distortion. These phosphatehydration-related differences in the vibrational spectrum of choline phospholipids are well known. ${ }^{48}$ The response of the symmetric phosphate band to dehydration is similar, although it shows a weaker shift from 1087 to $1094 \mathrm{~cm}^{-1}$. The shift of the phosphate stretching bands to higher frequencies upon dehydration is explained by the oxygen atoms becoming more free if their hydration decreases.

The $\mathrm{C}-\mathrm{O}-\mathrm{C}$ carboxylic acid ester bond connecting the fatty acids to the glycerol contributes an asymmetric stretch vibration around $1183 \mathrm{~cm}^{-1}$. In the hydrated state this band is broad and assumed to correspond to the superposition of the vibrations of the two bonds in the 1 and 2 positions. ${ }^{55}$ Upon dehydration the $\mathrm{C}-\mathrm{O}-\mathrm{C}$ stretching region experiences a shift toward lower frequencies $\left(1178 \mathrm{~cm}^{-1}\right)$ together with an increase in intensity and a narrowing. This is understood to be caused by a more dense packing of the lipids. The symmetric $\mathrm{C}-\mathrm{O}-\mathrm{C}$ stretch at $1065 \mathrm{~cm}^{-1}$ is less susceptible to dehydration.

Furthermore, in the dehydrated state characteristic wagging progressions of the aliphatic chains are observed, indicated by arrows in Fig. 11. The well resolved wagging vibrations at $1220 \mathrm{~cm}^{-1}$ and $1198 \mathrm{~cm}^{-1}$ in the dehydrated state refer to the alltrans conformation of the alkyl chains ${ }^{56,57}$ Upon hydration these wagging bands disappear, which is consistent with increasing disorder of the chain.

In the presence of PAH the following characteristic changes were observed: in the dehydrated state the asymmetric $\mathrm{PO}_{2}^{-}$ stretching vibration forms a broad band centered at $1232 \mathrm{~cm}^{-1}$, close to the original position in the hydrated state. A similar effect is observed for the symmetric stretch band, with no blue shift of the band upon dehydration.

These changes clearly point out to an interaction of PAH with the phosphate group of the lipid. PAH interacted with the phosphate group in such a way that prevented it from "dehydrating" but did not change its spectral characteristics at high water content. We interpret this effect as an attachment of the amino group of PAH to the phosphate of POPC via hydrogen bonding by replacing the originally bound water. ${ }^{58}$ The driving forces for the binding are most likely a combination of electrostatic attraction and the release of water from both the amino group and the phosphate, providing an entropic contribution to the process. This relatively strong interaction of phosphate ions with the amino groups of $\mathrm{PAH}^{59-62}$ can be easily demonstrated. When PAH is exposed to phosphate ions the aqueous solution becomes turbid, indicating phosphate-induced cross-linking of $\mathrm{PAH}$. The interaction with PAH in the phosphate region is also evident from the behavior of the $\mathrm{P}-\mathrm{O}(\mathrm{C})^{\mathrm{c}}$ stretching band at 1051 $\mathrm{cm}^{-1}$. As seen in the lower half of the figure, in the hydrated state this band is suppressed by PAH.

It is also likely that PAH induces changes in the $\mathrm{C}-\mathrm{O}-\mathrm{C}$ asymmetric stretching band upon dehydration. PAH did not affect the vibrational behavior of the carbonyl group (data not shown), and therefore hydrogen bonding of PAH to the carbonyl oxygen is unlikely. Details of the changes in the $\mathrm{C}-\mathrm{O}-\mathrm{C}$ region require a deeper analysis of the spectra, since there is an overlap of the $\mathrm{PAH} \mathrm{NH}_{3}{ }^{+}$rocking band ${ }^{63}$ with the lipid $\mathrm{C}-\mathrm{O}-\mathrm{C}$ stretching. This analysis will be the subject of further research, but it can be anticipated that the PAH effects on the headgroup are strong, as follows from the observation that the wagging 
progressions at dehydration disappear in the presence of $\mathrm{PAH}$. This behavior is consistent with a more disordered structure of the hydrophobic regions of POPC, indicating that the headgroups occupy a larger volume when interacting with $\mathrm{PAH}$.

\section{POPS-PAH}

Fig. 12 shows a section of the IR absorption spectrum of POPS taken in $\mathrm{D}_{2} \mathrm{O}$, covering the carbonyl stretching and the asymmetric carboxyl stretching bands in the absence (curve 1) and presence (curve 2) of PAH at high and low water content. Both the carbonyl stretching as well as the carboxyl stretching of POPS are sensitive to the hydration state. ${ }^{46-48}$ The carbonyl stretching region consists of two bands located at $1738 \mathrm{~cm}^{-1}$ and $1728 \mathrm{~cm}^{-1}$. Upon drying the band at $1728 \mathrm{~cm}^{-1}$ disappears, and is thus assigned to the hydrated state of the carbonyl group. ${ }^{46-48}$ The asymmetric carboxyl stretching, centered at $1623 \mathrm{~cm}^{-1}$ in the hydrated state, experiences upon dehydration a blue shift together with a broadening caused by increasing absorption at $1641 \mathrm{~cm}^{-1}$. This band is assigned to the dehydrated state of the carboxyl group.

In the presence of PAH the appearance of both bands changes considerably. The carbonyl stretching vibrations undergo a blue shift and there is a marked decrease in the band at $1728 \mathrm{~cm}^{-1}$, related to the hydrated state of the carbonyl oxygen. The conclusion was that PAH caused partial dehydration of the carbonyl oxygen in both chains, leading to a decrease of the hydrated fraction of the lipid at $1728 \mathrm{~cm}^{-1}$ both at low and high humidity. The effect of PAH on the carboxyl stretching was opposite. Here the interaction with PAH caused a red shift, indicating a strong interaction of the carboxyl group with PAH. The driving force for this interaction is the electrostatic attraction between the cationic PAH and the anionic carboxyl groups. From the comparatively strong effect on the vibrational spectrum it may be concluded that the amino groups of $\mathrm{PAH}$ are in direct contact with the $\mathrm{COO}^{-}$group rather than attracting it from a distance. It seems likely that the amino groups of PAH

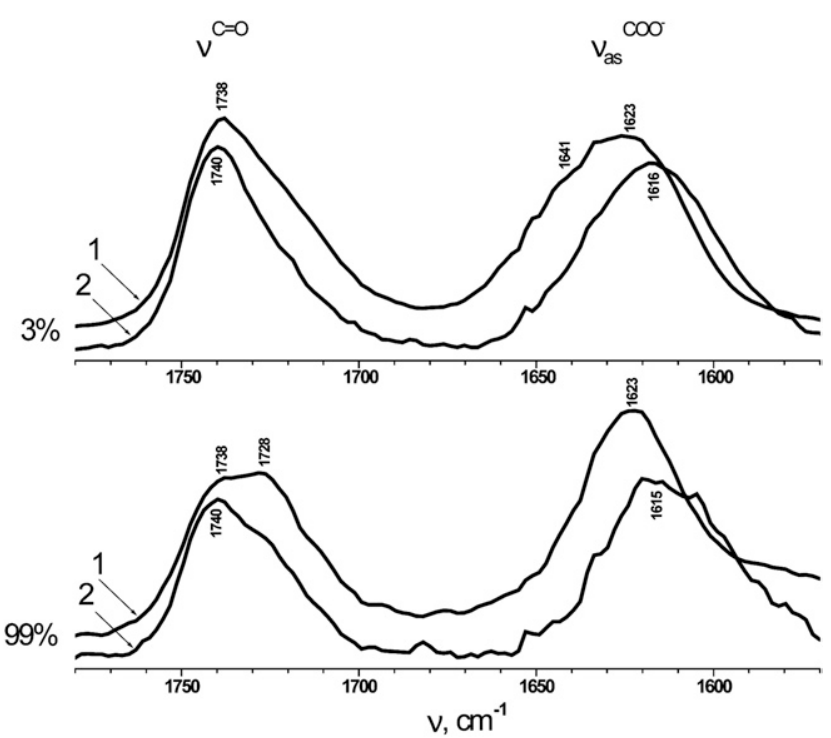

Fig. 12 FTIR spectra of hydrated (bottom) and dehydrated (top) pure POPS (curve 1) and POPS with PAH (curve 2) taken in $\mathrm{D}_{2} \mathrm{O}$. form hydrogen bonds with the oxygens of the carboxyl groups replacing bound water. The interaction of PAH with the phosphate group of POPS was very similar to that taking place in the case of POPC. The same is true for the PAH-induced inhibition of the wagging progressions in the dried state.

When comparing the interaction of PAH with POPS on one hand with POPC on the other hand, the infrared data leads to the conclusion that there are at least two points of attachment of PAH with POPS, namely the carboxyl and the phosphate moiety, while PAH binds to POPC only at the phosphate site. The $\mathrm{PAH}$ binding is accompanied by dehydration of these groups. A further important difference between POPS and POPC is that in the case of POPS the carbonyl oxygen of the fatty acid glycerol ester bonds is also dehydrated upon interaction with PAH.

\section{PDADMAC-POPS, PDADMAC-POPC}

In contrast to the interaction of POPS and POPC with PAH, where specific sites of interaction between the lipid headgroup and the polyelectrolyte were identified, the IR spectra of the lipids taken in the presence of PDADMAC did not reveal characteristic differences which could have been attributed to a specific interaction with the quarternary amino groups of PDADMAC. Only the carbonyl oxygen of POPS showed a weakly enhanced dehydration upon interaction with PDADMAC. The absence of spectral changes of both the phosphate and the carboxyl group of the lipids is understandable, since PDADMAC cannot participate in hydrogen bonding and thus can hardly change the spectral properties of the negatively charged sites of both lipids. The absence of IR spectral changes of the anionic sites when interacting with PDADMAC is therefore an indirect argument supporting the hypothesis that the amino group of PAH interacts with the anionic moieties of the phospholipids via hydrogen bonds replacing bound water, rather than only by electrostatic attraction.

A deeper analysis of the $\mathrm{OH}$ stretching region of water in the presence of PDADMAC showed that the role of adsorbed water may be the key for understanding the strong interaction of PDADMAC with POPS and POPC. This is demonstrated in Fig. 13, which displays the stretching vibrational region of water

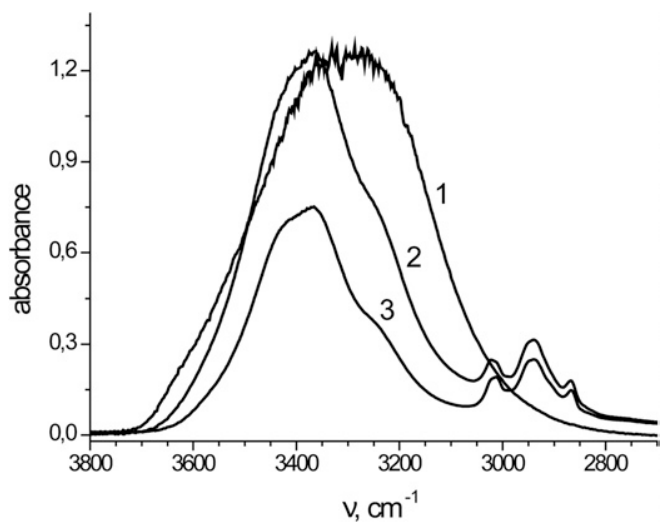

Fig. 13 IR absorbance in the $\mathrm{H}_{2} \mathrm{O}$ stretching region of PDADMAC (curve 2) and PDADMAC + POPS (curve 3 ) at a relative humidity of $30 \%$. The control (curve 1) shows pure water. Water associated with POPS has been subtracted in curve 3 . 
ranging from approximately $3600 \mathrm{~cm}^{-1}$ to $3100 \mathrm{~cm}^{-}$. Curve 2 shows the $\mathrm{H}_{2} \mathrm{O}$ spectrum in the presence of PDADMAC at a humidity of $30 \%$. The control in curve 1 shows the IR absorption of pure $\mathrm{H}_{2} \mathrm{O}$. The differences between the two spectra are striking. The absorption range in the presence of PDADMAC is comparatively narrow compared to the control, and is also remarkably blue shifted. The absorbance around $3200 \mathrm{~cm}^{-1}$ is significantly depressed, indicating a decrease of the "coupledin-phase" symmetric water stretching vibrations. From this data it can be inferred that the water in the proximity of PDADMAC is decoupled. This is consistent with the presence of more weakly interacting water molecules in the proximity of the polymer chain due to the unfavorable topology.

Curve 3 in Fig. 13 shows the absorbance of the PDADMACassociated water in the presence of POPS. While POPS did not influence the qualitative spectral characteristics of PDADMACassociated water, the data shows that the amount of water was greatly diminished. At a humidity of $30 \%$, about one third of the PDADMAC-associated water was released upon interaction with POPS. Consequently, it may be concluded that one of the driving forces of the PDADMAC-lipid self-assembly is the release of water molecules in contact with the PDADMAC chain. In the case of POPS the electrostatic attraction between the overall negatively charged headgroup and the quarternary ammonium group will be an additional driving factor for interaction, with adsorption of POPS causing the release of anions from the PDADMAC chain.

\section{Conclusions}

The formation of SLBs involves at least three distinct steps. The initial adhesion of the vesicles is followed by spreading and simultaneous stress-induced rupture of the lipid layers. Finally, adjacent lipid patches fuse and form a continuous lipid bilayer. For attachment and rupture of the lipid vesicles, the affinity towards the support should be large enough, whereas the formation of the continuous membrane requires local rearrangements of the lipid coverage, for which some lateral mobility of the lipids along the surface of the support is an essential condition. The formation of continuous lipid bilayers on solid supports from adsorbing vesicles is thus only possible by a subtle balance of the involved forces. If attraction of lipids toward the support is too strong, the resulting small lateral mobility may not allow for local rearrangements of the lipid coverage, which are necessary to form a continuous bilayer. The formed lipid layer may lack connectivity or have large defects. On the other hand, if the attractive forces are too weak, vesicle deformation is limited and the process of layer formation is arrested at the stage of vesicle adsorption, since spreading and rupture do not occur.

If the support is a polyelectrolyte multilayer, it has to be kept in mind that such a structure is nothing like a solid surface, but rather characterized by a certain softness and fuzziness at the interface. Instead of, for example, deformation of the adsorbed vesicles toward a solid surface upon attraction, the polyelectrolyte molecules themselves may be partially pulled out from the multilayer and form an adsorbed layer onto the lipid vesicles in the region of contact. Which of these two scenarios takes place will depend on both the magnitude of the involved forces and the time scales of the ongoing processes. For example, vesicle deformation is largely elastic and thus basically a fast process, with the possible rate-limiting step of volume reduction requiring the formation of transient pores or permeation of the enclosed solution through the membrane. On the other hand, reorganization of the polyelectrolyte multilayer may extend over longer time scales, but does not require large forces. The softness of a polyelectrolyte multilayer may, however, vary considerably depending on layer composition and ionic conditions, which have a pronounced influence on the interaction between polyanions and polycations.

The transition of adsorbed vesicles towards a supported bilayer on a soft polyelectrolyte support is a complex process involving a number of steps. There are, moreover, many degrees of freedom. A large set of parameters including lipid and ionic composition, mechanical and chemical properties of the multilayer, and the sequence of handling have an influence on the final result. It appears thus unlikely that there are simple rules allowing prediction of whether a continuous lipid bilayer will be formed or if interaction will result into one topology or the other. This conclusion is consistent with the results of the present work, where we show that very different structures, ranging from genuine bilayers to adsorbed vesicles and novel 3D lipid-polyelectrolyte phases on colloidal particles, may be formed by varying the lipid and polyelectrolyte layer composition, as well as the ionic conditions.

One of the most unexpected results concerning the formation of lipid layers on polyelectrolyte multilayer supports was that neither POPC nor POPS alone formed a proper bilayer on PAH as the top layer, while a $1: 1$ mixture of both lipids formed a lipid bilayer both on colloids and on flat macroscopic surfaces. Fig. 3, Fig. 5, and Fig. 8 illustrate this result in a most convincing way. How can this apparent contradiction be explained? Let us consider first the interaction of POPC with a PSS/PAH multilayer. The obtained results reveal that the layer formation process is stopped at the vesicle adsorption step. Spreading and rupture, let alone fusion did not take place. We attribute this behavior basically to the absence of long-range attractive forces between the zwitterionic POPC and the positively charged PAH. Although the binding of PAH to the phosphate group of POPC is strong enough, it does not create sufficiently large bending forces on the vesicle membrane. The side chains of the PAH bind to the phosphate group if they come in close contact, but the entropic contraction of the polymer chain is obviously too weak to induce spreading. It is conceivable that in this case PAH may form an adsorption layer on the vesicle by slowly rearranging the multilayer structure. This view is supported by the adsorption of POPC vesicles on the PAH terminated multilayer, which continues over a time frame of hours indicating the slowly ongoing rebuilding of the multilayer. Even if PSS was the top layer, the PAH located underneath was responsible for the adsorption of POPC vesicles as was concluded from the results presented in Fig. 4. The similarities in adsorption of POPC regardless of whether PAH or PSS were the outermost layers indicate that rebuilding processes of the multilayer finally result in similar architectures.

There is a rather strong attraction of POPS to PAH driven by both electrostatic long-range forces and subsequent short range binding to the carboxyl and phosphate groups via hydrogen bonds. This leads to spreading of adsorbed vesicles, but the lipid 
may not be able to slide on the multilayer surface because it becomes too strongly bound. The result would be a patchy coverage by POPS, but the formation of a connective bilayer is inhibited. Small defects where the PAH surface is exposed to the bulk may serve as attachment sites for incoming POPS vesicles, which, however, do not experience spreading forces as the initially adsorbing ones, since they are attached only at small focal areas. This scenario is consistent with the QCM data, the topology as shown in Fig. 6, and the observed limited diffusion.

The $1: 1$ mixture of POPC and POPS showed a seemingly perfect behavior in terms of spreading and fusion. This is, in our view, the result of a subtle balance of the involved forces and kinetics. POPS is responsible for the long-range attraction and thus is the key to vesicle spreading and rupture. The presence of POPC weakens the overall attraction and is thus essential for some remaining capability for motion along the surface, leading to a connective lipid bilayer. Furthermore, vesicles formed from the mixture may have a reduced stability toward bending stress, which would result in rupture already before the rearrangement processes of the multilayer architecture cause a decrease in the induced stress.

A relevant feature of these PEM-supported lipid bilayers is that the lipid molecules in direct contact with the polyelectrolyte support are immobilized because of the short range interactions between the amino groups of PAH and the phospholipid headgroups. In this sense, these structures are essentially different from natural systems such as biological membranes.

Further research is clearly necessary to fully understand the mechanisms outlined here. For example, it seems feasible to independently measure the development of a connective layer by applying fluorescence-based fusion assays in a time-resolved manner through flow cytometry. The rate of vesicle adsorption may also be quite important in determining the resulting structure. Experiments focusing on this aspect can be performed with QCM or flow cytometry.

It is difficult from the results of only one characterization technique to conclude on the quality of the formed layer. Our earlier work on lipid deposition on polyelectrolyte structures may have resulted in less perfect layers than originally assumed on the basis of the employed characterization techniques. ${ }^{64}$ The same may be true for other data, in view of the comparatively narrow window in the parameter space which is open for the formation of a lipid bilayer from vesicle adsorption on soft surfaces.

We have found that the amino groups of PAH play a special role in binding to phospholipids. PAH-induced dehydration of the phosphate and the carboxyl group of the lipids, which may be an important aspect when the biological effects of polyamines such as peptides or antimicrobial agents are concerned.

\section{Acknowledgements}

This work was supported by a grant from the Deutsche Forschungsgemeinschaft (DFG) DO 410/4-1. L. Cuéllar is a fellow of CONACYT, Mexican Council of Science. S.E. Moya is a Ramón y Cajal fellow and thanks this program and a grant (MAT 2007-60458) from the Spanish Ministry of Education and Science for support.

\section{References}

1 G. Storm and D. J. A Crommelin, Pharm. Sci. Technol. Today, 1998, 1, 19.

2 E. Sackmann and M. Tanaka, Trends Biotechnol., 2000, 18, 58.

3 R. P. Richter, J. Lai Kee Him and A. Brisson, Mater. Today, 2003, 6, 32.

4 M. J. Grogan, Y. Kaizuka, R. M. Conrad, J. T. Groves and C. R. Bertozzi, J. Am. Chem. Soc., 2005, 127, 14383.

5 J. T. Groves and M. L. Dustin, J. Immunol. Methods, 2003, 278, 19.

6 E. M. Winter and J. T. Groves, Anal. Chem., 2006, 78, 174.

7 B. Cannon, N. Weaver, Q. Pu, V. Thiagarajan, S. Liu, J. Huang, M. W. Vaughn and K. H. Cheng, Langmuir, 2005, 21, 9666.

8 T. Yang, S.-Y. Jung, H. Mao and P. S. Cremer, Anal. Chem., 2001, 73, 165.

9 A. Loidl-Stahlhofen, J. Schmitt, J. Nöller, T. Hartmann, H. Brodowsky, W. Schmitt and J. Keldenich, Adv. Mater., 2001, 13, 1829.

10 I. Geissbuehler, R. Hovius, K. L. Martinez, M. Adrian, R. Thampi and H. Vogel, Angew. Chem., Int. Ed., 2005, 44, 1388.

11 M. Fischlechner, U. Reibetanz, M. Zaulig, D. Enderlein, J. Romanova, S. Leporatti, S. Moya and E. Donath, Nano Lett., 2007, 7, 3540.

12 R. P. Richter, R. Bérat and A. R. Brisson, Langmuir, 2006, 22, 3497.

13 J. M. Johnson, T. Ha, S. Chu and S. G. Boxer, Biophys. J., 2002, 83, 3371.

14 K. Dimitrievski and B. Kasemo, Langmuir, 2008, 24, 4077.

15 C. G. Sinn, M. Antonietti and R. Dimova, Colloids Surf., A, 2006, 282-283, 410.

16 C. G. Sinn, R. Dimova and M. Antonietti, Macromolecules, 2004, 37, 3444.

17 C. G. Sinn, R. Dimora, C. Huin, O. Sel and M. Antonietti, Macromolecules, 2006, 39, 6310.

18 L. Toellner, M. Fischlechner, B. Ferko, R. M. Grabherr and E. Donath, Clin. Chem. (Washington, D. C.), 2006, 52, 1575.

19 A. S. Angelatos, B. Radt and F. Caruso, J. Phys. Chem. B, 2005, 109, 3071.

20 A. S. Angelatos, K. Katagiri and F. Caruso, Soft Matter, 2006, 2, 18.

21 R. Georgieva, S. Moya, S. Leporatti, B. Neu, H. Bäumler, C. Reichle, E. Donath and H. Möhwald, Langmuir, 2000, 16, 7075.

22 R. Georgieva, S. E. Moya, H. Bäumler, H. Möhwald and E. Donath, J. Phys. Chem. B, 2005, 109, 18025.

23 G. Krishna, T. Shutava and Y. Lvov, Chem. Commun., 2005, 22, 2796.

24 S. E. Moya, R. Georgieva, H. Bäumler, W. Richter and E. Donath, Med. Biol. Eng. Comput., 2003, 41, 504.

25 S. Moya, E. Donath, G. B. Sukhorukov, M. Auch, H. Bäumler, H. Lichtenfeld and H. Möhwald, Macromolecules, 2000, 33, 4538.

26 A.-L. Troutier and C. Ladavière, Adv. Colloid Interface Sci., 2007, 133,1 .

27 G. Köhler, S. E. Moya, S. Leporatti, C. Bitterlich and E. Donath, Eur. Biophys. J., 2007, 36, 337.

28 A. P. R. Johnston, A. N. Zelikin, L. Lee and F. Caruso, Anal. Chem., 2006, 78, 5913.

29 M. A. Frias, S. B. Diaz, N. M. Ale, A. Ben Altabef and E. A. Disalvo, Biochim. Biophys. Acta, Biomembr., 2006, 1758, 1823.

30 C. Delajon, T. Gutberlet, R. Steitz, H. Möhwald and R. Krastev, Langmuir, 2005, 21, 8509.

31 H. Boroudjerdi, Y.-W. Kim, A. Naji, R. R. Netz, X. Schlagberger and A. Serr, Phys. Rep., 2005, 416, 129.

32 M. Fischlechner, L. Toellner, P. Messner, R. Grabherr and E. Donath, Angew. Chem., Int. Ed., 2006, 45, 784.

33 M. Antonietti and C. Göltner, Angew. Chem., Int. Ed. Engl., 1997, 36, 910.

34 M. Antonietti, A. Kaul and A. Thünemann, Langmuir, 1995, 2633.

35 M. Antonietti and A. Wenzel, Colloids Surf., A, 1998, 135, 141.

36 N. Kohli, S. Vaidya, R. Y. Ofoli, R. M. Worden and I. Lee, J. Colloid Interface Sci., 2006, 301, 461.

37 R. Kügler and W. Knoll, Bioelectrochemistry, 2002, 56, 175.

38 T. Cassier, A. Sinner, A. Offenhäuser and H. Möhwald, Colloids Surf., B, 1999, 15, 215.

39 N. Kahya and P. Schwille, J. Fluoresc., 2006, 16, 671.

40 L. Ramos, M. Schönhoff, Y. Luan, H. Möhwald and G. Brezesinski, Colloids Surf., A, 2007, 303, 79.

41 S. Moya, W. Richter, S. Leporatti, H. Bäumler and E. Donath, Biomacromolecules, 2003, 4, 808 . 
42 A.-M. Pilbat, Z. Szegletes, Z. Kóta, V. Ball, P. Schaaf, J.-C. Voegel and B. Szalontai, Langmuir, 2007, 23, 8236.

43 D. Hoekstra, T. de Boer, K. Klappe and J. Wilschut, Biochemistry, 1984, 23, 5675.

44 Y. Luan and L. Ramos, J. Am. Chem. Soc., 2007, 129, 14619.

45 S. Hong, P. R. Leroueil, E. K. Janus, J. L. Peters, M.-M. Kober, M. T. Islam, B. G. Orr, J. R. Baker and M. M. Banaszak Holl, Bioconjugate Chem., 2006, 17, 728.

46 A. Blume, W. Hübner and G. Messner, Biochemistry, 1988, 27, 8239

47 W. Hübner and A. Blume, Chem. Phys. Lipids, 1998, 96, 99.

48 P. Garidel, A. Blume and W. Hübner, Biochim. Biophys. Acta, Biomembr., 2000, 1466, 245.

49 A. V. Popova and D. K. Hincha, Biophys. J., 2003, 85, 1682.

50 H. Binder, T. Gutberlet, A. Anikin and C. V. Klose, Biophys. J., 1998 , 74, 1908.

51 W. Hübner and A. Blume, Chem. Phys. Lipids, 1998, 96, 99.

52 H. Binder, G. Köhler, K. Arnold and O. Zschörnig, Phys. Chem Chem. Phys., 2000, 2, 4615.

53 H. Binder, K. Arnold, A. S. Ulrich and O. Zschörnig, Biophys. Chem., $2001,90,57$
54 H. L. Casal, H. H. Mantsch, F. Paltauf and H. Hauser, Biochim. Biophys. Acta, 1987, 919, 275.

55 H. Binder, Appl. Spectrosc. Rev., 2003, 38, 15.

56 H. L. Casal and H. H. Mantsch, Biochim. Biophys. Acta, 1984, 779, 381.

57 L. Senak, D. Moore and R. Mendelsohn, J. Phys. Chem., 1992, 96, 2749.

58 K. Lutz, C. Gröger, M. Sumper and E. Brunner, Phys. Chem. Chem. Phys., 2005, 7, 2812.

59 D. Kovacevic, S. van der Burgh, A. de Keizer and M. A. Cohen Stuart, J. Phys. Chem. B, 2003, 107, 7998.

60 D. R. Kioussis, F. W. Wheaton and P. Kofinas, Aquat. Eng., 2000, 23, 315.

61 D. R. Kioussis, F. W. Wheaton and P. Kofinas, Aquat. Eng., 1999, 19, 163.

62 S. S. Shiratori and M. F. Rubner, Macromolecules, 2000, 33, 4213.

63 B. J. M. Rajkumar and V. Ramakrishnan, J. Raman Spectrosc., 2000, 31, 1107.

64 M. Fischlechner, O. Zschörnig, J. Hofmann and E. Donath, Angew. Chem., Int. Ed., 2005, 44, 2892. 